\title{
Study of the Doppler Broadening of Positron Annihilation Radiation in Silicon
}

\author{
E. do Nascimento, O. Helene, V. R. Vanin, \\ Instituto de Física, Universidade de São Paulo, C.P. 66318, 05315-970, São Paulo, SP, Brazil \\ and M. Moralles \\ Instituto de Pesquisas Energéticas e Nucleares - IPEN, C.P. 11049, 05422-970, São Paulo, SP, Brazil
}

Received on 15 July, 2005

\begin{abstract}
We report the measurement of Doppler broadening annihilation radiation in silicon, using ${ }^{22} \mathrm{Na}$ as a positron source, and two Ge detectors arrangement. The two-dimensional coincidence energy spectrum was fitted using a model function. The model function included at rest positron annihilation with valence band, $2 \mathrm{p}, 2 \mathrm{~s}$, and $1 \mathrm{~s}$ electrons. In-flight positron annihilation was also fitted. The detectors response functions included backscattering, and a combination of Compton effects, pileup, ballistic deficit, and pulse shaping problems. The obtained results agree well with the literature.
\end{abstract}

\section{INTRODUCTION}

Doppler broadening study of positron-electron annihilation radiation is an important tool in the field of materials science $[1,2]$. Usually, the results of Doppler broadening experiments have been analyzed through the comparison of the calculated annihilation probability density with the experimental data. In this work we went in the inverse direction: a model function was fitted to the experimental data in order to obtain the distribution of electron momenta, similar to the analysis accomplished for the aluminum [3,4]. In these works the coincidence energy spectrum was fitted using a model function, accounting for both Doppler broadening and detector system response. Intensities of the thermalized positron annihilation with band, 2p, 2s, and 1s electrons, and in-flight positron annihilation were fitted. The binding energies of the $1 \mathrm{~s}, 2 \mathrm{~s}$, and $2 p$ electrons and the Fermi cutoff parameters of the band electrons were also fitted. This procedure allows the experimental determination of the annihilation parameters and response function parameters with their uncertainties and the $\chi^{2}$-test of the obtained results.

\section{EXPERIMENTAL SETUP}

The two annihilation gamma-rays energies $E_{1}$ and $E_{2}$ were measured using two Ge detectors forming an angle of $180^{\circ}$ with each other and separated by $5 \mathrm{~cm}$. A $3.7 \times 10^{5} \mathrm{~Bq}(10$ $\mu \mathrm{Ci}){ }^{22} \mathrm{Na}$ source was placed between two $2 \mathrm{~mm}$ thick silicon mono-crystal sheets (Czochralski). Previous lifetime measurements results obtained were 219.0 ps with $97.7 \%$ of intensity, 473 ps with $2.0 \%$ of intensity, and 2800 ps with $0.3 \%$ of intensity. An ${ }^{192}$ Ir source was simultaneously measured in order to calibrate the detectors and to follow any energy calibration drift during the experiment. ${ }^{60} \mathrm{Co}$ and ${ }^{137} \mathrm{Cs}$ sources were also measured. A two dimensional spectrum was taken for about $730 \mathrm{~h}$. The two dimensional histogram in the $E_{1}, E_{2}$ plane in the region of interest is presented in (Figure 1).

This spectrum of Figure 1 can be interpreted as follows. The crest along the line $E_{1}+E_{2}=1022 \mathrm{keV}$ is mainly due to at rest positron annihilation with core and band electrons. The ridges parallel to the axes are due to the coincidence between an annihilation gamma-ray and a Compton scattered gammaray (either the other annihilation radiation or the $1274.5 \mathrm{keV}$ gamma-ray from ${ }^{22} \mathrm{Na}$ decay). When in-flight positron annihilates with a low momentum electron, two gamma rays are emitted. Near the $511 \mathrm{keV}-511 \mathrm{keV}$ peak in the two dimensional spectrum this annihilation can be approximated by a crest along a circular arc function centered at $E_{1}=E_{2}=3 / 2 m c^{2}$ [4]. This curve can be barely seen in (Figure 1).

\section{FITTING - FUNCTION MODEL}

To describe quantitatively the measured spectrum, a two dimensional function was fitted to the experimental histogram in an $87 \mathrm{keV}$ x $87 \mathrm{keV}$ region around the two photon annihilation peak. Positron annihilation with valence band was represented by

$$
f_{v}=\sum_{i=1}^{2} C_{i}\left(E_{1}-E_{2}-\alpha_{i}\right)\left(E_{1}-E_{2}+\alpha_{i}\right)+\frac{A_{v} e^{\frac{-\left(E_{1}-E_{2}\right)^{2}}{2 \sigma_{v}^{2}}}}{\sqrt{2 \pi} \sigma_{v}}
$$

along the line $E_{1}+E_{2}+B_{v}=1022 \mathrm{keV}$, where $B_{v}$ is the gap energy of the silicon [5], $E_{1}$ and $E_{2}$ are energies in detectors 1 and 2 respectively, $\alpha_{i}$ are the cutoff parameters $\left(C_{i}=0\right.$ when $\left.\left|E_{1}-E_{2}\right|>\alpha_{i}\right)$. The parabolas were used in the fitting because they fitted better to the experimental data. Positron annihilation with $2 \mathrm{p}$ electrons was fitted by

$$
f_{2 p}=\frac{A_{2 p} e^{\frac{-\left(E_{1}-E_{2}\right)^{2}}{2 \sigma_{2 p}^{2}}}}{\sqrt{2 \pi} \sigma_{2 p}}
$$

along the line $E_{1}+E_{2}+B_{2 p}=1022 \mathrm{keV}$, where $B_{2 p}$ is the $2 \mathrm{p}$ electron binding energy [6]. Positron annihilation with $2 \mathrm{~s}$ electrons was fitted by

$$
f_{2 s}=\frac{A_{2 s} e^{\frac{-\left(E_{1}-E_{2}\right)^{2}}{2 \sigma_{2 s}^{2}}}}{\sqrt{2 \pi} \sigma_{2 s}}
$$


along the line $E_{1}+E_{2}+B_{2 s}=1022 \mathrm{keV}$, where $B_{2 s}$ is the 2 s electron binding energy [6]. Positron annihilation with $1 \mathrm{~s}$ electrons was fitted by

$$
f_{1 s}=\frac{A_{1 s} e^{\frac{-\left(E_{1}-E_{2}\right)^{2}}{2 \sigma_{1 s}^{2}}}}{\sqrt{2 \pi} \sigma_{1 s}}
$$

along the line $E_{1}+E_{2}+B_{1 s}=1022 \mathrm{keV}$, where $B_{1 s}$ is the $1 \mathrm{~s}$ electron binding energy [6]. Finally, when $\left(E_{1}, E_{2}\right)$ is a point inside the circle centered at $\left(3 m_{0} c^{2} / 2,3 m_{0} c^{2} / 2\right)$, a function given by

$$
f_{f}=A_{f} e^{-\lambda d}
$$

where

$$
d=\frac{m_{0} c^{2}}{2}-\sqrt{\left(E_{1}-3 m_{0} c^{2} / 2\right)^{2}+\left(E_{2}-3 m_{0} c^{2} / 2\right)^{2}}
$$

is the distance from $\left(3 m_{0} c^{2} / 2,3 m_{0} c^{2} / 2\right)$ to $\left(E_{1}, E_{2}\right)$, and was used to take into account the in-flight positron annihilation. Finally, two internal $\left(E<E_{g}\right)$ and two external $\left(E>E_{g}\right)$ exponential queues were included in order to simulate the nonGaussian part of the detectors response functions. Two internal and two external ridges along the lines $E_{1}=511 \mathrm{keV}$ and $E_{2}=511 \mathrm{keV}$, proportional to the peak intensity, were included in the fit. The background in channel (i,j) was empirically considered as proportional to the product of the total number of counts (peak excluded) along the lines $j=$ constant by the total number of counts along the line $\mathrm{i}=$ constant; the single fitted parameter was the proportionality factor. The backscattering was described by a parabola, along the line $E_{1}+E_{2}=1022 \mathrm{keV}$ and centered at $511 \mathrm{keV}-511 \mathrm{keV}$. Functions $f_{1 s, 2 s, 2 p, v}$ and the four exponential queues, after summing, were convoluted with the detector response functions given by two Gaussians. The fitted parameters were $A_{1 s}, A_{2 s}, A_{2 p}, A_{v}$, $\sigma_{1 s}, \sigma_{2 s}, \sigma_{2 p}, \sigma_{v}, C_{1,2}, \alpha_{1,2}, a_{1,2}$, the background parameter, the peak positions in the two energy axes, the angular inclination of the $E_{1}+E_{2}=1022 \mathrm{keV}$ line respect to the main axes, the sixteen exponential queues parameters (eight amplitudes and eight attenuation factors), the detector resolutions, and four parameters for the ridges intensities. Differently from ref. [4], the electron binding energies were not fitted. The fit was done by using the least-squeares method. The GaussMarquardt method was used to consider the non-linear parameters [7],[8]. The chi-squared value was calculated by

$$
\chi^{2}=\sum_{i, j} \frac{\left(n_{i j}-F_{i j}\right)^{2}}{F_{i j}}
$$

where $n_{i j}$ is the number of observed events in channel $(i, j)$ of the two dimensional spectrum (Figure 2), and $F_{i j}$ is the fitted function (Figure 3).

\section{RESULTS AND CONCLUSION}

The obtained results to the core ( $2 \mathrm{p}, 2 \mathrm{~s}$, and $1 \mathrm{~s}$ electrons) and valence band annihilation intensities were $2.27(3) \%$ and

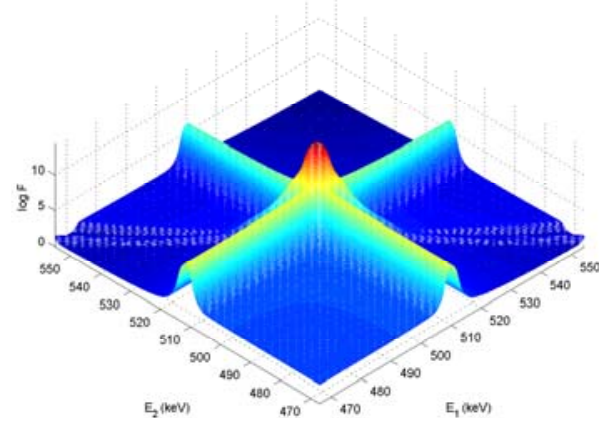

FIG. 1: The two-dimensional representation for the electron-positron annihilation peak, in this figure the in-flight annihilation coincidence is represented.

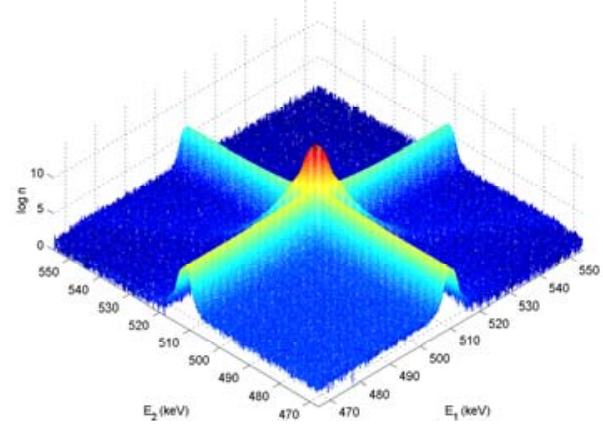

FIG. 2: Experimental two-dimensional spectrum of the annihilation gamma-rays.

97.73(3)\%, respectively. The intensities obtained of literature [9] to the core and valence band were $2 \%$ and $98 \%$, respectively. We have found that a complete analysis of the twodimensional Doppler annihilation radiation spectrum, in the case of the silicon, is possible. Unlike the usual approach, this procedure allows the determination of the data uncertainties. Thus, hypotheses can be tested and different results can be averaged.

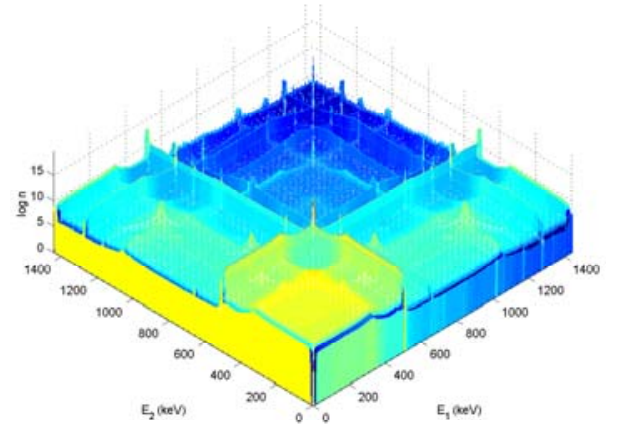

FIG. 3: Fitted two-dimensional spectrum of annihilation gammarays. 


\section{Acknowledgments}

We wish to acknowledge the support of Conselho Nacional de Desenvolvimento Científico e Tecnológico - CNPq and
Fundação de Amparo à Pesquisa do Estado de São Paulo, and the help of Dr. M. C. A. Fantini.

\section{REFERENCE}

[1] K G Lynn, J R MacDonald, R A Boie, L C Feldman, J D Gabbe, M F Robbins, E Bonderup, and J Golovchenko, Phys Rev Lett 38, 241 (1977).

[2] A W Hunt, D B Cassidy, F A Selim, R Haakenaasen, T E Cowan, R H Howell, K G Lynn, and J A Golovchenko, Nucl Instr and Meth B (2000) 44.

[3] E do Nascimento, O Helene, V R Vanin, and C Takiya, Braz J Phys 34, 1017 (2004).

[4] E do Nascimento, O Helene, C Takiya, and V R Vanin, Nucl Instr
Meth A 538, 723 (2005).

[5] N W Ashcroft, N D Mernin (1976) Solid State Physics, Saunders College Publishing, USA

[6] R B Firestone and V S Shirley, Table of Isotopes, eighth edition, vol II, John Willey \& Son, (1996)

[7] V. R. Vanin, G. Kenchian, M. Moralles, O. A. M. Helene, and P. R. Pascholati, Nucl Instr Meth A 391, 338 (1997).

[8] D. W. Marquardt, J Soc Appll Math 11, 431 (1963).

[9] M Hakala, M J Puska, and R M Nieminen, Phys Rev B 57, 7621 (1998). 